\title{
Towards personalised positive psychiatry?
}

\section{Commentary on "Vantage sensitivity: a framework for individual differences in response to psychological intervention"}

\author{
Boris Klingenberg ${ }^{1} \cdot$ Bart P. F. Rutten ${ }^{1}$
}

Received: 19 May 2018 / Accepted: 30 July 2018 / Published online: 14 August 2018

(c) Springer-Verlag GmbH Germany, part of Springer Nature 2018

\section{Introduction}

In their paper entitled "Vantage sensitivity: a framework for individual differences in response to psychological intervention" de Villiers et al. highlight the importance of environmental sensitivity as an overarching meta-framework and emphasize studying the concept of 'vantage sensitivity'. While environmental sensitivity refers to differences between individuals in how pronounced they respond to (positive and negative) stimuli, the concept of vantage sensitivity focusses strictly on exposure to positive stimuli and its concurrent response. In a comprehensive fashion, the authors discuss possible genetic, physiological and psychological factors that may underlie vantage sensitivity and they furthermore propose the implementation of the Highly Sensitive Person (HSP) scale in adults and the Highly Sensitive Child (HSC) scale in children as part of psychometric workups.

Whilst the manuscript provides a valuable addition to the field through its summary, review, discussion and suggestions for future research, it also raises a number of questions, such as (1) whether (and to which extent) exposures can be qualified as positive or negative, (2) to which extent does the presented data on vantage sensitivity overlap with other concepts and phenotypes, for instance the placebo-effect, i.e., what is the specificity of vantage sensitivity?

This comment refers to the article available at https://doi. org/10.1007/s00127-017-1471-0.

Bart P. F. Rutten

b.rutten@maastrichtuniversity.nl

1 Department of Psychiatry and Neuropsychology, School for Mental Health and Neuroscience (MHeNs), European Graduate School of Neuroscience (EURON), Maastricht University Medical Centre, P.O. Box 5800, 6202 AZ Maastricht, The Netherlands

\section{Classifying exposures}

The concept of vantage sensitivity revolves around the response to positive exposures, and it partially contrasts the concept of differential susceptibility, which comprises the response to positive and negative exposures. While some robust risk (i.e., increasing risk) and protective (i.e., decreasing risk) factors have been identified for a range of mental disorders, the majority of environmental exposures cannot easily be classified or dichotomised into 'positive' or 'negative'. To clarify the notion of mixed exposures and in keeping with the main focus of the review on psychotherapy, some examples of the downsides of psychotherapy might illustrate this point. In summary, Berk and Parker report that roughly $10 \%$ of patients worsen after commencing psychotherapy; furthermore there is a potential increase in dependency as well as a possible risk of increasing rumination in overly rational cognitive behavioural therapies [1].

Furthermore, it may be that the impact of environmental exposures varies across developmental stages in life, and between individuals. An environmental exposure during one specific moment in life, or in one specific context may promote well-being, while it decreases well-being or even increases risk for pathology during other phases or in another contexts in life. For instance, it is shown in a rat model for PTSD that resting phase sleep deprivation, with or without forced wakefulness, diminished the expression of the PTSD-like phenotype [2]. Yet broadly speaking, sleep deprivation is usually not a good thing. In human subjects, an interesting finding by Harding and Mezulis is that rumination may represent both a protective and a risk factor for depressive symptoms [3].

In addition, it should be noted that a risk factor for one individual, may be a neutral or a protective factor for others. Grabe et al. show for instance a GxExE model that shows a 
synergistic effect of childhood and adult trauma depending on 5-HTTLPR genotype [4].

\section{Pleiotropy}

The authors describe a list of genetic variations which may be related to vantage sensitivity. These genetic variations have popped up in numerous genetic studies and have been linked with a range of mental phenotypes $[5,6]$. Yet, there does not seem to be a clear specificity in the effects of the selected genes. Take the serotonin transporter gene polymorphism (5-HTTLPR) for instance, its function has been associated with a whole plethora of psychiatric phenotypes ranging from the better documented role in major depressive disorder to obsessive compulsive disorder; as well as harm avoidance and resilience [7,8]. A comparable pattern emerges for other gene variants and phenotypes. For instance, the review of Hall et al. on genetic variation underlying the placebo response reported an interesting list of associated genes and SNPs [9]. It mentions the usual suspects of serotonin polymorphisms (TPH2, SLC6A4, HTR2A, 5-HTTLPR) and dopamine polymorphisms (COMT, MAO-A, DBH, DRD3, BDNF) as well as the opioid receptor and endocannabinoid receptor, i.e., some of the same variants as described to be possibly linked with vantage sensitivity.

The arrival of genome-wide association studies further underlines the above problem of aspecificity of genetic polymorphisms. As the authors point out, a constructed polygenic score, with more than 20,000 gene variants, regarding the response to cognitive behavioural therapy showed that subjects with a higher genetic sensitivity score showed an increased response to higher quality CBT. Although interesting, it should be noted that genetic studies in mental health phenotypes have not (yet) provided clear causal pathways, and that there are "many associated SNPs of each small effect" associated with different mental phenotypes or underlying latent phenotypes.

Resilience concerns itself mostly with the differential response in mental well-being of people when confronted with adversity and how some people swiftly recover from adversity and may even show the capacity for posttraumatic growth [10]. The latter part of this definition starts to edge into the territory of vantage sensitivity. It might be hypothesised that parts of the subdivisions of the meta-framework of environmental sensitivity also overlap in the contribution of the underlying neurobiology [11]. In resilience research there has been additional evidence that individual adaptation is the resulting effect of GxE interactions particularly in the stress response system [10], e.g., with carriers of genetic variants in CRH1 and FKBP5 (impacting on the response of the hypothalamus-pituitary axis) having lower risk to develop mental ill-health in response to adversity. Evidence suggests that secure attachment seems to play a more central role in how resilience is shaped by environmental effects. Interesting findings from behavioural genetic studies with twins are that attachment indeed is the solitary result of environmental influences. Sensitive and warm parenting particularly appears to be a central determinant of attachment security as it is the parents who provide the child with a safe and stimulating learning environment from which it can explore the world. Internal working models are formed by securely attached children in which they perceive themselves as worthy, others as being available and reliable, and the environment can be experienced as challenging but manageable with support from others. Working models like these lead to improved self-regulation, the possibility to infer own and other's mental states, to handle social relationships and to effectively deal with various stressors. This reasoning closely follows some of the implications the authors point out. In summary, the authors reason that there is some preliminary research that indicates that vantage sensitivity builds over time, according to positive exposures. They give the example of vagal tone, an index of autonomic flexibility, which seems to be in a mutually reinforcing relationship with positive emotions and social connectedness. The measurement for social connectedness scored questions like: "During the social interactions, I felt "in tune" with the person/s around me". This seems to tap into the prosocial component and the adult dimensions of attachment as we sketched earlier. Thus, different facets of environmental sensitivity seem to be interlinked.

\section{Discussion}

Although it is necessary to establish some post signs in the landscape of causality, the primary concern of the authors seems to be a very clinically relevant problem: not everyone responds to (psycho-) therapy in the same manner. As the authors suggest, it might be worthwhile to focus on validating a refined psychometric workup of which the HSP/HSC could be a part. Fine-tuning could be aimed at the mentioned big 5 personality facets of low extraversion and high conscientiousness as well as perhaps a weighting based on some baseline demographics as age, sex, severity, chronicity, comorbidity, social support and clinician/treatment contextual factors. Such an approach does need to be combined with prospective study approaches capturing environmental (social and biological) exposures, when possible using objective physiological and biological phenotypes as well as self-reports on appraisal and experiences in the flow of daily life [12] and clinical interview-based mental phenotypes, allowing analyses of longitudinal changes over time in dynamic, context-sensitive network models. Such 
approaches are required in order to better describe and interpret interindividual variations in response to environmental factors in a life span perspective.

When using such a psychometric workup inevitably patients will present themselves who are highly motivated yet are predicted to have a diminished response to psychotherapy. The authors suggest the possibility of changing treatment-modality, -intensity or duration in these cases. They reframe it into: "being less sensitive to one type of treatment may not necessarily mean lacking sensitivity to all treatments". In this line, one could furthermore argue that sensitivity to treatment depends on social context and expectations of the individual, and thus that lack of treatment response at a certain time point in life does not necessarily predict lack of response at another time point in life.

Indeed, scientific endeavours on answering who may benefit from treatment (and when) are currently ongoing for a range of treatment modalities. Such initiatives should consider incorporating the concept of vantage sensitivity as another soldier within the ranks of positive psychiatry, while underlining the importance of personalised treatment.

\section{Compliance with ethical standards}

Conflict of interest On behalf of all authors, the corresponding author states that there is no conflict of interest.

\section{References}

1. Berk M, Parker G (2009) The elephant on the couch: side-effects of psychotherapy. Aust N Z J Psychiatry 43(9):787-794
2. Cohen S, Kaplan Z, Zohar J et al (2017) Preventing sleep on the first resting phase following a traumatic event attenuates anxietyrelated responses. Behav Brain Res 320:450-456

3. Harding KA, Mezulis A (2017) Is rumination a risk and a protective factor? Eur J Psychol 13(1):28-46

4. Grabe HJ, Schwahn C, Mahler J et al (2012) Moderation of adult depression by the serotonin transporter promoter variant (5-HTTLPR), childhood abuse and adult traumatic events in a general population sample. Am J Med Genet B Neuropsychiatr Genet 159B(3):298-309

5. Burmeister M, McInnis MG, Zöllner S (2008) Psychiatric genetics: progress amid controversy. Nat Rev Genet 9(7):527-540

6. Sullivan PF, Daly MJ, O'Donovan M (2012) Genetic architectures of psychiatric disorders: the emerging picture and its implications. Nat Rev Genet 13(8):537-551

7. Sinopoli VM, Burton CL, Kronenberg S et al (2017) A review of the role of serotonin system genes in obsessive-compulsive disorder. Neurosci Biobehav Rev 80:372-381

8. Ebstein RP (2006) The molecular genetic architecture of human personality: beyond self-report questionnaires. Mol Psychiatry 11(5):427-445

9. Hall KT, Loscalzo J, Kaptchuk TJ (2015) Genetics and the placebo effect: the placebome. Trends Mol Med 21(5):285-294

10. Rutten BPF, Hammels C, Geschwind N et al (2013) Resilience in mental health: linking psychological and neurobiological perspectives. Acta Psychiatr Scand 128(1):3-20

11. Boyce WT (2016) Differential susceptibility of the developing brain to contextual adversity and stress. Neuropsychopharmacology 41(1):142-162

12. van Os J, Verhagen S, Marsman A et al (2017) The experience sampling method as an mHealth tool to support self-monitoring, self-insight, and personalized health care in clinical practice. Depress Anxiety 34(6):481-493 